\title{
A Note on Primality Testing Using Lucas Sequences
}

\author{
By Michael A. Morrison \\ Dedicated to D. H. Lehmer on his 70 th birthday
}

\begin{abstract}
For an odd integer $N>1$, thought to be prime, a test is given which uses Lucas sequences and which can establish that any prime divisors of $N$ are $\equiv \pm 1$ modulo the factored portion of $N+1$.
\end{abstract}

For an odd integer $N>1$, the complete factorization of $N+1$ or $N-1$ provides sufficient information to establish whether or not $N$ is prime (see [1], [2]). Unfortunately, when $N$ is large, it is generally very difficult and time-consuming to complete such a factorization. In such a case, the partial factorization can be extremely useful, since it can be used to restrict any possible divisors of $N$ to a small number of arithmetic sequences with (hopefully) large differences.

The theory of Lucas sequences becomes useful when considering $N+1$. A theorem of D. H. Lehmer [1] asserts that, if $p$ is a prime such that $p^{\alpha} \| N+1$, and if there exists a Lucas sequence with certain properties, then any divisor of $N$ is of the form $a p^{\alpha} \pm 1$. Thus, if $s$ distinct primes were known to divide $N+1$, there would be $2^{s}$ different sequences which might contain a factor of $N$. The following theorem shows that it is possible to reduce these $2^{s}$ sequences to only two: namely, $\{a P+1\}$ and $\{a P-1\}$, where $P$ is the factored portion of $N+1$. THEOREM. Let $D$ be an integer such that the Jacobi symbol $(D / N)=-1$, and let $N+1=R \Pi_{i=1}^{s} p_{i}^{\alpha_{i}}$ where, for all $i, p_{i}$ is prime and $\left(R, p_{i}\right)=1$. If for each $i$ there exists a Lucas sequence $\left\{U_{k}^{(i)}\right\}$ with discriminant $D$ such that

$$
N \mid U_{N+1}^{(i)}
$$

and

$$
\left(U_{(N+1) / p_{i}}^{(i)}, N\right)=1,
$$

then every prime divisor $n$ of $N$ satisfies $n \equiv \pm 1 \quad\left(\bmod \Pi_{i=1}^{s} p_{i}^{\alpha_{i}}\right)$.

Proof. Let $n$ be a prime divisor of $N$ and let $\omega_{i}(n)$ denote its rank of apparition in $\left\{U_{k}^{(i)}\right\}$. Then $n \mid U_{k}^{(i)}$ if and only if $\omega_{i}(n) \mid k$, and thus (1) implies that for each $i, \omega_{i}(n)$ exists and divides $N+1$. But (2) implies that $\omega_{i}(n) \nmid(N+1) / p_{i}$, and thus $p_{i}^{\alpha_{i}} \mid \omega_{i}(n)$ for each $i$.

Received July 12, 1974.

AMS (MOS) subject classifications (1970). Primary 10A25; Secondary 10A35.

Key words and phrases. Primality testing, Lucas sequences. 
However, since $n$ is prime, $n \mid U_{n-(D / n)}^{(i)}$. Hence, $\omega_{i}(n) \mid n-(D / n)$ for each $i$, which implies that $p_{i}^{\alpha_{i}} \mid n-(D / n)$ for each $i$. Therefore, $n \equiv(D / n)= \pm 1\left(\bmod \Pi_{i=1}^{s} p_{i}^{\alpha_{i}}\right)$. $\pm 1\left(\bmod \Pi_{i=1}^{s} p_{i}^{\alpha_{i}}\right)$.

Department of Mathematics University of California

Los Angeles, California 90024

1. D. H. LEHMER, “An extended theory of Lucas functions," Ann. of Math., v. 31, 1930, pp. $442-443$.

2. E. LUCAS, "Théorie des fonctions numériques simplement périodiques," Amer. J. Math., v. 1,1878 , p. 302 . 